Glasplatte mit Aluminiumfolie beklebt, die geerdet wurde. Vor der beklebten Seite der Platte war eine Koronasonde beweglich angebracht, so daß die Bewegung jedes Punktes der Platte gemessen werden konnte. Die beim Schwingen der Platte an der Sonde auftretende Wechselspannung wurde verstärkt und einem Oszillographen zugeführt. Die Platte wurde durch das Antriebssystem eines Lautsprechers punktförmig erregt (s. Abb. 28). Die Versuchsanordnung wurde in der Weise geeicht, daß an den Plattenrän- dern die Amplitude der Schwingung sowohl optisch mit dem Mikroskop als auch mit der Koronasonde bestimmt wurde.

So konnten die Schwingungsformen der Platte bei verschiedenen Anregungsbedingungen bestimmt werden. Für den Fall, daß die Platte an einer äußeren Ecke erregt wurde, kam sie bei einer aufgedrückten Frequenz von $120 \mathrm{~Hz}$ in Resonanz. Die ermittelte Schwingungsform gibt Abb. 29 wieder. Aufgezeichnet sind die Kurven konstanter Amplitude.

\title{
Zum Elementarvorgang der Lichtbeugung
}

\author{
Von W. Kossel und K. Strohmaier \\ Aus dem Physikalischen Institut der Universität Tübingen \\ (Z. Naturforschg. 6 a, 504-508 [1951]; eingegangen am 13. August 1951)
}

\begin{abstract}
Es wird an die verschiedenen Auffassungen der Beugung erinnert und darauf hingewiesen, daß die Beugung des Lichts an einem durchsichtigen Schirm einen engeren Vergleich mit derjenigen von Röntgenstrahlen oder Materiewellen an Kristallgittern erlaubt als der gewohnte Fall des undurchlässigen Schirmes. Versuche über die Beugung an einer Kante eines durchsichtigen Schirmes werden dargelegt und in einigen Photometerkurven der Intensitätsverlauf wiedergegeben. Von den Einzelheiten wird als wesentlich die Methode der Markierung des gecmetrischen Schattens der Kante im Strahlengang angegeben.
\end{abstract}

1.

S eit den ersten theoretischen Deutungen der Beugung gibt es zwei grundsätzlich verschiedene Auffassungen von der Herkunft der Erscheinungen. Thomas Young versuchte die Beugung an einer Kante als eine Überlagerung der ungestörten Wellenbewegung und einer Welle, die von der materiellen Kante ausgeht, zu deuten. Dagegen baut Fre s n el seine Beugungstheorie auf das $\mathrm{H}$ u y gen s sche Prinzip auf, er betrachtet nur den durch materielle Grenzen eingeschlossenen freien Raum der Beugungsöffnung als Ausgangspunkt der Sekundärwellen. Diese zweite Betrachtungsweise wurde dann lange bevorzugt. 1917 endlich bewies Rubinowicz ${ }^{1}$, an Sommerfelds strenge Behandlung der Randbeugung am undurchlässigen Schirm anknüpfend, die funktionentheoretische Äquivalenz der Fresnelschen und der Youngschen Auffassung. Die letztere wird dabei um eine charakteristische Angabe über die Phasenlage der Randwelle ergänzt.

Diese formale Einsicht traf sich aufs beste mit neuartigen experimentellen Aufgaben. Als v. La u e 1912 die Beugung der Röntgenstrahlen an Kristallen ent-

1 A. Rubinowicz, Ann. Physik 53, 257 [1917]. deckte, leitete ihn der Gedanke, daß die Elektronenwolken der Materie die Sekundärwellen aussenden. Dieser Gedanke ist für die Röntgeninterferenzen bestimmend geblieben - sein anschaulicher Ausdruck sind die Atomformfaktoren. Ebenso ist für Elektronenund Neutronenbeugung die Materie der Ausgangspunkt der Sekundärwellen. Alles das zusammen mit dem Anblick der leuchtenden Kante aus dem Dunkelraum macht deutlich, daß es auch beim Licht schließlich die Atome der die beugende Öffnung begrenzenden Materie sind, mit denen das Licht beim Durchgang in Wechselwirkung tritt. Eine mit der Behandlung der Röntgenwellen übereinstimmende Darstellung muß in ihnen, nicht im leeren Raum, die Ausgangspunkte für die charakteristische Lichterscheinung erblicken.

\section{2.}

Von hier aus gesehen, sind die gewohnten Fälle der Beugung an Kanten und Öffnungen undurchlässiger Schirme, die in allen klassischen Untersuchungen betrachtet werden, offenbar ungünstig. Die Theorie behandelt die Materie mittels Grenzbedingungen, über deren Inhalt und Berechtigung die Diskussion nie ganz zur Ruhe kommt, und das Experiment zeigt - wir erinnern an die bekannten Aufnahmen 
Arkadiews ${ }^{2}-$, daß die Eigenschaften der Materie, auch der Radius der beugenden Kante, die Beugungserscheinung kaum beeinflussen. Um das Mitspielen der Materie ausgiebig zur Geltung kommen zu lassen, sollte sie vielmehr von einer kräftigen Lichtbewegung durchsetzt, und die von hier ausgehenden Bewegungen sollten bestimmend an der Beugungserscheinung beteiligt werden. Das heißt: man verwende durchsichtige Schirme. Am besten würden homogene Schirme von konstanter Dicke sein. Sie schalten in die fortlaufende Bewegung eine konstante Phasenverzögerung ein. So wird die Beugung an ihnen zur Beugung an „Phasenkanten“.

An dieser Beugung an einer Phasenkante sind außerdem auch ganz andere aktuelle Untersuchungen interessiert: einmal hängt davon ab, wie weit die Abbildung mittels Phasenkontrasts die Struktur des Objekts wiedergibt, zum anderen hat die Elektronenmikroskopie stark damit zu tun, einerseits wiederum wegen der Objekttreue der Abbildung, andrerseits weil aus Beugungserscheinungen auf das Auflösungsvermögen geschlossen werden kann. Zum dritten hat die Beugung an einer Phasenkante kürzlich durch Wolte $r^{3}$ in seiner Minimalstrahlkennzeichnung eine experimentell überaus brauchbare Anwendung gefunden.

3.

Da man dies Endergebnis der Vorgänge, die Phasenveränderung, gern so fertig hinnimmt, betonen wir mit einigen Worten, daß sie ein Mitspielen der Materie von ganz derselben Art bedeutet wie bei der Röntgenstreuung. Daß die normale Phasenverzögerung beim Durchsetzen durchsichtigen $\mathrm{Ma}$ terials - das Phänomen der Lichtbrechung - durch immer erneute Anregung sekundärer Quellen in den Raumteilen des Materials zustande kommt, also völlig den in der Röntgen- und Elektronenoptik „dynamisch" genannten fortlaufenden Umbildungen der Welle im Material entspricht, wird schon in den klassischen Dispersionstheorien zugrunde gelegt und läßt sich leicht auch für den Elementarunterricht lebendig machen ${ }^{4}$. Um das gleiche auch an den bekannten Interferenzerscheinungen eines Blättchens klar zu sehen, denke man sich die Wirkung eines homogenen durchsichtigen Blattes der Dicke $D$ auf folgendem Wege aufgebaut. Man teile es (Abb. 1) in zur Blättchen- und zur Einfallsebene normale Schichten, die so dünn sind, daß die ihrer Dicke zuzuordnende

2 W. A rka di e w, Physik. Z. 14, 832 [1913].

3 H. W ol t e r, Z. Naturforschg. 5 a, 139 [1950].

4 W. K o s s e l, Optik 3, 190 [1948].
Phasenänderung zu vernachlässigen ist. Die Gesamtstreuung des in einer solchen Schicht enthaltenen Materials baut sich also ebenso auf, wie die eines schräg angeleuchteten Spaltes der Breite $D$ nach dern Huygensschen Prinzip aufgebaut wird. Auf bekannten Rechenwegen - etwa der zuerst von Rubinowicz gegebenen Integration - ergibt sich, daß die resultierende Erregung außerhalb des Primärbündels aus zwei den Rändern zuzuordnenden Erregungen aufgebaut gedacht werden kann, die gegeneinander um $180^{\circ}$ verschoben sind. Das obere und das untere Ende des Streifens $\left(E_{0}\right.$ und $\left.E_{\mathrm{u}}\right)$ wirken also als Zentren resultierender Sekundärwellen, die bei Fraunhofer-Beobachtung - in einer gegen die Dicke $D$ großen Entfernung - so in Paare zusammenzufassen sind, wie Abb. 1 andeutet.

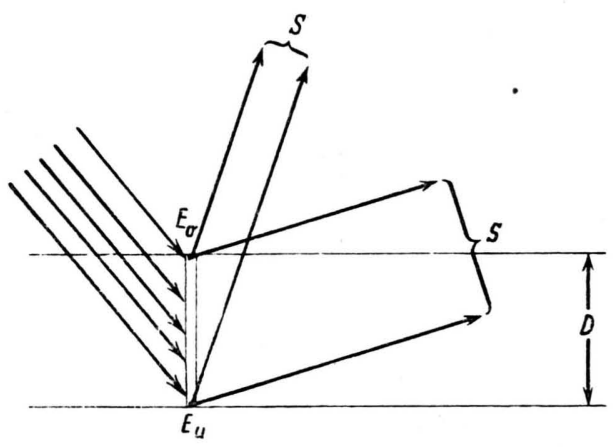

Abb. 1. Zur Ableitung der optischen Gesamtwirkung einer durchsichtigen Platte aus den Streuwirkungen der Volumelemente.

Die Gesamtwirkung der Schicht wird weiter aufgebaut, indem man die Wirkungen der benachbarten Schichten summiert. Da die maßgebenden Schichtenden miteinander zwei Ebenen bilden, faßt man zunächst die Sekundärbewegungen der oberen Enden für sich zusammen: sie unterstützen einander nur in der einen Richtung, in der die Einfallsebene beibehalten wird und der Winkel gegen die Ebene der Sekundärzentren dem Einfallswinkel auf dieser Ebene gleich ist. So wird aus der Gesamtstreuung der Schichten der ganzen Platte eine „Spiegelung“ an ihrer oberen und unteren Grenzebene.

$\mathrm{Da}$ diese beiden Randbewegungen einander um $180^{\circ}$ entgegenwirken, folgt hier aus dem bekannten Ersatz des Spalts durch seine Ränder, aus dem Satz, der die Youngsche Randbewegung aus der Fresnelschen Summierung über die Spaltbreite wiederherstellt. Die Aussage, daß die in den Schattenraum gebeugte Randwelle um $180^{\circ}$ verschoben sei gegen die in den hellen Raum laufende, ergibt hier die 


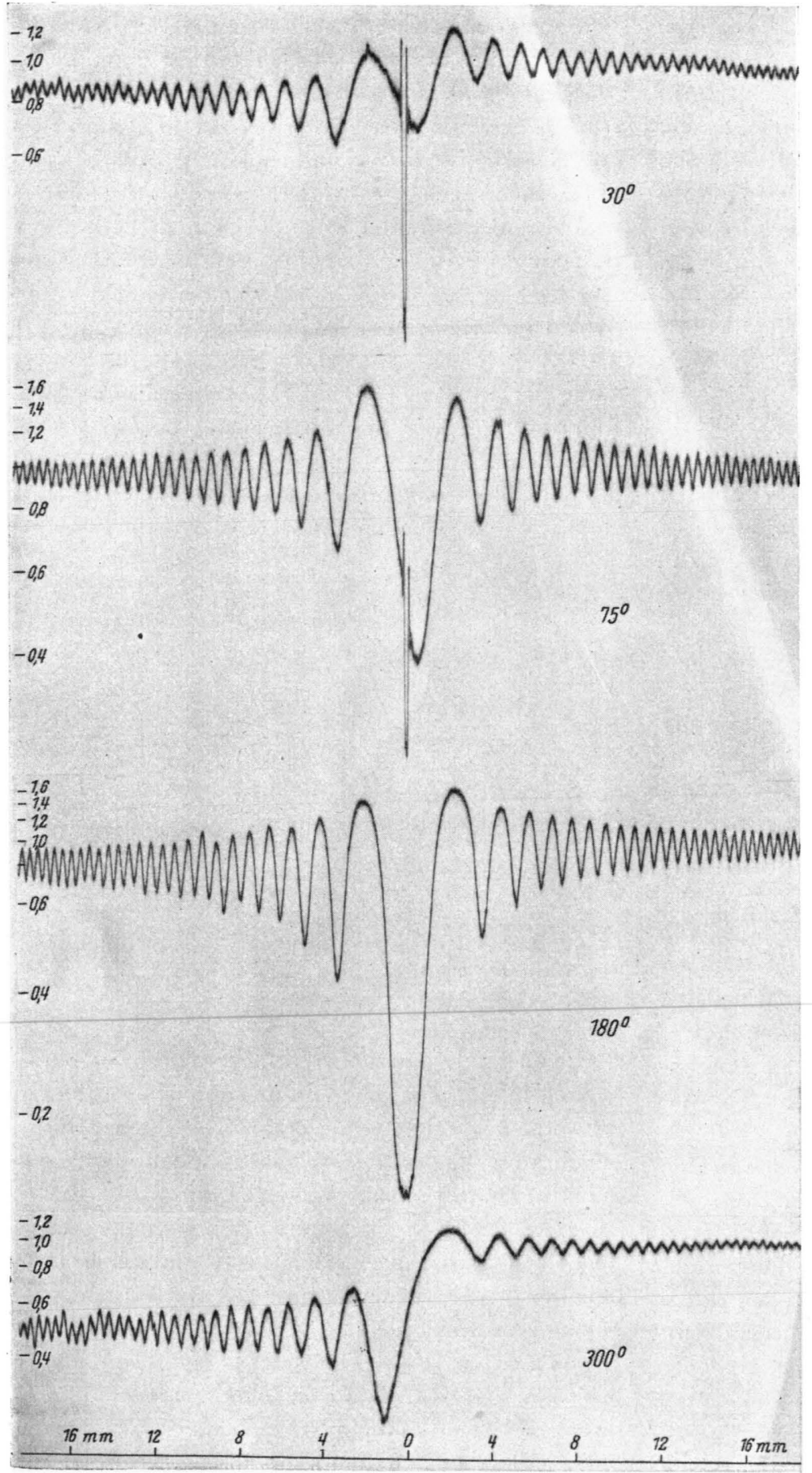

Abb. 2. Beugung an Phasenkanten wachsenden Gangunterschiedes. Unveränderte Photometerkurven, Intensivitätsskala nach Stufenkeil links angegeben. Die beiden obersten Bilder mit Marke des als Null der Lagenskala geltenden geometrischen Schattens der Kante. $\lambda=5461 \AA$, Abstand Spalt - Kante $=100 \mathrm{~cm}$, Kante - Platte $=300 \mathrm{~cm}$.

Phasendifferenz zwischen Spiegelung am optisch dichteren und Spiegelung am dünneren Medium, die man aus den Randbedingungen der Maxwell-Theorie herzuleiten pflegt. Spiegelung an Grenzflächen ist das Resultat der Integration über die Streuwirkungen des an die Grenzfläche anstoßenden Materials.

Die Summierung wird vollendet, indem man in der bekannten Weise nach dem Zusammenwirken der oberen und der unteren Spiegelung fragt. Mehrfache Spiegelung bedeutet erneute Streuung der Sekundärbewegungen, also vollständigen „dynamischen " Vorgang. So wird über die Gesamtbewegung entschieden, die die von Streuzentren erfüllte Platte etwa nach rückwärts sendet - es folgen die elementaren Überlegungen über Farben dünner Blättchen, speziell etwa über Reflexionsvernichtung bei bestimmten Dicken.

\section{4.}

Es war uns erwünscht, über diesen Vorgang der Beugung an der Phasenkante genaue quantitative Daten zu besitzen. Vorläufige Versuche (Danzig 1S44) hatten gezeigt, daß alles auf Anwendung eines homogenen Schirmmaterials ankommt. Wie dies mit Hilfe der anodischen Oxydation von Aluminium, bei der ja der Vorgang selbst auf Gleichförmigkeit der Dicke hinarbeitet, hergestellt wurde, berichtet die folgende Notiz - das so gewonnene Material ist auch für andere Anwendungen sehr brauchbar. Von einer Kontrolle der Brechungsindizes auf einem von diesen Versuchen unabhängigen Wege berichtet die ihr folgende Mitteilung von Herrn Dr. Weis kirchner.

Wir schildern hier die Grundzüge der Beobachtungsmethode und ihre Ergebnisse. Ausführlicher soll, da sich gezeigt hat, daß die Beobachtungen einer weiteren Verschärfung wert sind, später berichtet werden.

Gefiltertes Quecksilberlicht der Wellenlänge 5461 $\AA$ tritt durch einen $25 \mu$ breiten, $2 \mathrm{~mm}$ hohen Spalt in 
einen $4 \mathrm{~m}$ langen Strahlengang, trifft in den hier wiedergegebenen Beispielen nach $1 \mathrm{~m}$ die Blättchenkante, nach weiteren $3 \mathrm{~m}$ die Beobachtungsebene der Lupe oder die photographische Platte. Derartige Aufnahmen der Fresnel-Beugung sind in Abb. 2 wiedergegeben.

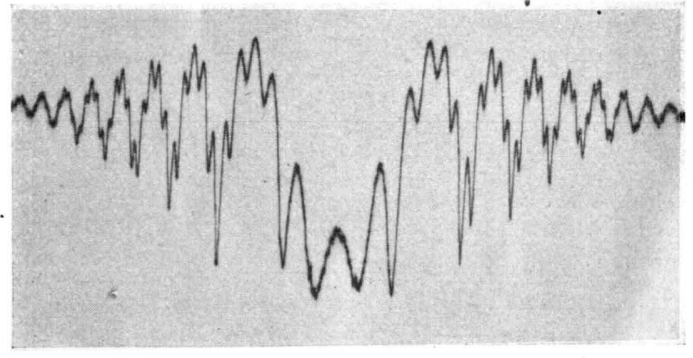

Abb. 3. Beispiel zur Justierung des Strahlenganges. gehende Vertikalebene fixiert. Aus der Justierung daran, die Schritt um Schritt geschah, gibt Abb. 3 ein Beispiel: der Spalt ist bereits vertikal gestellt, es geht nun darum, von ihm aus eine gerade Linie festzulegen. In 30 und in $100 \mathrm{~cm}$ Abstand von ihm sind Lote aufgehängt, deren Beugungsbilder sich überlagern. An der Symmetrie dieses Gesamtbildes läßt sich sehr scharf die Geradlinigkeit der Strecke vom Spalt über die beiden Lote einstellen. Das Beispiel der Abb. 3 zeigt eine noch unvollständige Einstellung; die hier sichtbare Dissymmetrie entspricht einer $\mathrm{Ab}$ weichung von $10 \mu$. Weiterhin wird die Blättchenkante justiert. Auf den Hauptaufnahmen ist schließlich jeweils das Beugungsbildchen eines nahe vor der Platte hängenden Lotes enthalten. Als feine Lücke und Zwickenspur gibt es in der Photometerkurve die Lage des geometrischen Schattens der Blättchenkante.

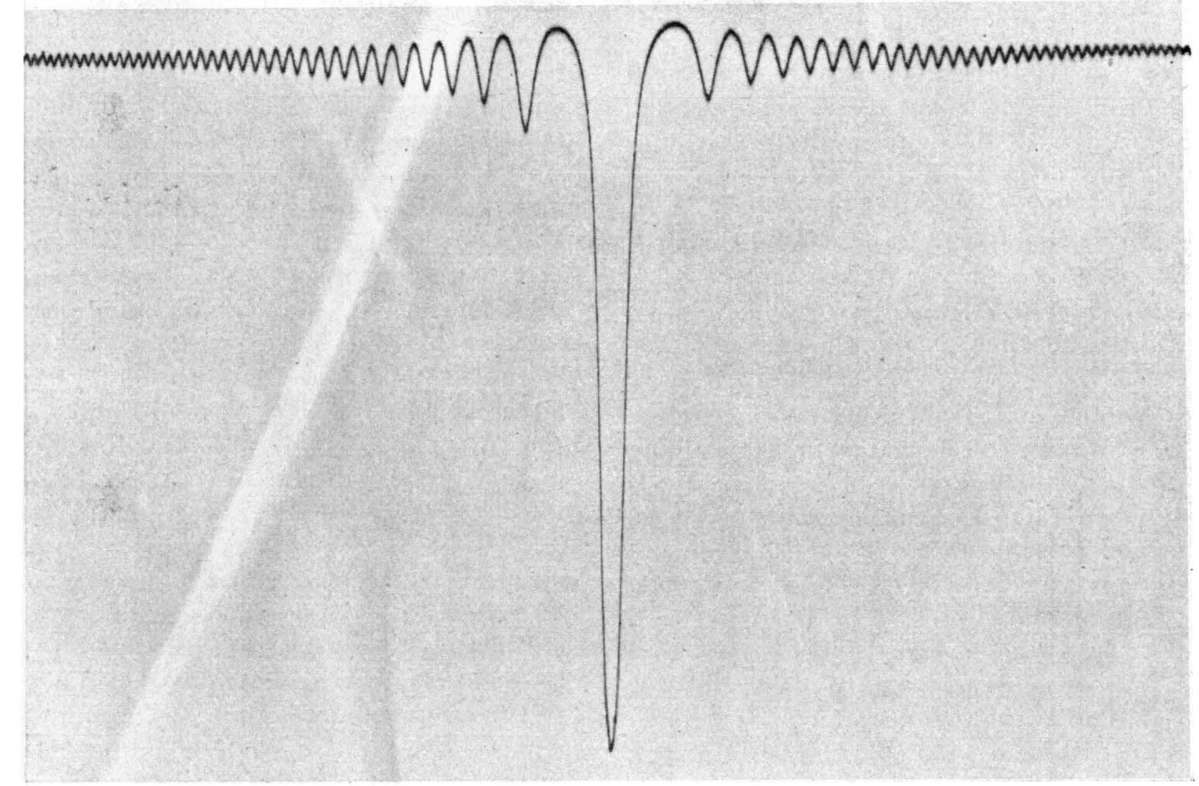

Abb. 4. Dritter Fall von Abb. 2 in längerer Exposition, um das bei $180^{\circ}$ eintretende Verschwinden der Intensität im Minimum vor Augen zu führen (Fall der „Minimumstrahlkennzeichnung“ nach H. W o lt e r 3 ).

Es kommt darauf an, als Anhaltspunkt in jedem Bild die Richtung des geometrischen Schattens zu fixieren. Über den Strahlengang hinweg läuft von Zimmerwand zu Zimmerwand eine $6 \mathrm{~m}$ lange Stahlsaite von $150 \mu$ Stärke, die mit $1 \mathrm{~kg}$ gespannt ist. An ihr hängen verschiebbare Lote aus Haar von $50 \mu$ Stärke in den Strahlengang hinein, die unten mit 10-g-Gewichtchen in mit Öl gefüllte Dämpfungsgefäße hineinreichen, die von Tischchen auf der Dreikantschiene getragen werden. So ist eine durch-
Das an der undurchsichtigen Kante nur im freien Raum auftretende Streifenbild ist nun nach beiden Seiten entwickelt. Das Mittelminimum, das zunächst auf der Schattenseite liegt, wandert mit wachsender optischer Dicke des Blättchens nach außen, erreicht mit der Phasenverschiebung um $180^{\circ}$ die Linie des geometrischen Schattens und rückt weiter nach außen. Bei $180^{\circ}$ geht es zu verschwindender Intensität herunter: dies ist der von Herrn Wolter ausführlich behandelte Fall der "Minimumstrahlkennzeichnung“. 
Abb. 4 stellt ihn in stark exponierter Aufnahme gesondert dar.

Von einer Phasenverschiebung von $30^{\circ}$ an sind Aufnahmen in Schritten von $15^{\circ}$ ausgeführt. Abb. 2 zeigt die erste von ihnen, dann mit $75^{\circ}$ nahezu eine der optischen Dicken, an denen die Reflexion verschwindet - also in diesem Bild der durchgehenden Bewegung beide Seiten wieder gleich hell erscheinen
—, dann den $180^{\circ}$-Fall und einen Zustand nahe der Verzögerung um eine Vollwelle mit wohlausgebildetem Streifensystem und dem wieder abgeflachten Hauptminimum.

Eingehende Daten über den Intensitätsverlauf sollen erst nach Einsatz der vollen heutigen Erfahrungen, wie sie z. B. die beiden folgenden Notizen bringen, mitgeteilt werden.

\section{NOTIZEN}

\section{Ein einfaches Verfahren zur Herstellung großfl̈̈chiger durchsichtiger Häute aus Aluminiumoxyd mit einer Dicke zwischen 50 und $300 \mathrm{~m} \mu$}

\author{
Von $\mathrm{K}$ ar l Stro hma i e r
}

Physikalisches Institut der Universität Tübingen

(Z. Naturforschg. 6 a, 508-509 [1951]; eingeg. am 13. Aug. 1951)

$\mathrm{Zu}$ bestimmten physikalischen Untersuchungen sind dünne Häute der oben angegebenen Dicke notwendig. Für diese Zwecke wurden bislang hauptsächlich Kollodiumhäute verwendet, die etwa nach dem Verfahren von Trenktrog, wie es v. Angerer ${ }^{1}$ beschreibt, hergestellt wurden. Leider sind diese thermisch und chemisch weder so widerstandsfähig noch von so konstanter Dicke, wie es oft wünschenswert wäre. In diesen Fällen können die nachfolgend beschriebenen Häute zu besseren Ergebnissen führen.

Die Oxydhaut wird anodisch auf Aluminium erzeugt. Die Methoden hierzu sind seit langem zur Herstellung von Elektrolyt-Kondensatoren entwickelt worden. $\mathrm{H}$ a $\beta$ und $\mathrm{Kehler}{ }^{2}$ haben diese Oxydschichten erstmals in Form von freitragenden Häutchen als Trägerfolien in der Elektronenmikroskopie verwendet. Dabei gingen sie von aufgedampftem Aluminium aus. v. Ardenne und Friedrich-Freks a ${ }^{3}$ gehen zum selben Zwecke von Aluminiumfolien aus, und $\mathrm{Walkenhorst}{ }^{4}$ gibt für solche Schichten an, daß bei der Verwendung von 3-proz. Ammoniumcitrat als Elektrolyt ein Dickenzuwachs von $13,7 \AA /$ Volt eintritt. In den beiden zuletzt genannten Arbeiten wurde das Aluminium mit Sublimatlösung entfernt. Dies geht leicht bei kleinen Flächen, wie sie hauptsächlich als Trägerfolie Verwendung finden. Bei größeren Flächen zerstören die sich bildenden Gasblasen sehr leicht die dünne Haut. Außerdem bereitet es Schwierigkeiten, Flächen von einigen Quadratzentimetern auf einen Rah'men zu bringen und aus der Flüssigkeit zu heben. Diese

1 W. Tre n k tro g, Diss. Kiel 1923, wiedergegeben in v. A n g e r e r, Technische Kunstgriffe bei physikalischen Untersuchungen, 6. Aufl. Vieweg, Braunschweig 1944, S. 117 .

2 G. H a ß u. H. K e h le r, Kolloid-Z. 95, 26 [1941].

3 M. v. Ardenne u. H. Friedrich-Freksa, Naturwiss. 29, 523 [1941].

4 W. W a l k e $\mathrm{n}$ h or st, Naturwiss. 34, 373 [1947].
Schwierigkeiten lassen sich leicht auf die nachfolgend beschriebene Weise umgehen.

Als Ausgangsmaterial dient eine $10-15 \mu$ starke Folie aus Reinaluminium* , die gegen das Licht gehalten keine Poren aufweisen darf und etwas größer als clas benötigte Fenster aus Aluminiumoxyd sein soll. Sie wird zunächst auf einer sauberen, staubfreien Glasplatte mit einem weichen Tuch geglättet. Mit einer Klammer, die gleichzeitig die elektrische Zuleitung darstellt, wird sie gefaßt und etwa $1 \mathrm{~cm}$ tief senkrecht in die Elektrolytflüssigkeit eingehängt und bei ungefähr $160 \mathrm{~V}$ oxydiert. Als Kathode kann ebenfalls ein Streifen aus Aluminium oder auch Kohle verwendet werden. Der Anfangsstrom wird durch einen Vorwiderstand auf etwa 0,3 A begrenzt. Löst sich beim Oxydieren das Aluminium stellenweise auf, so ist der Elektrolyt verunreinigt und unbrauchbar. Nach 2 bis 3 Min. ist die Oxydation beendet, und der oxydierte Streifen kann in dest. Wasser gespült werden. Auf dieselbe Weise deckt man auch die übrigen drei Ränder auf beiden Seiten mit Oxyd ab. Die so vorbereitete und getrocknete Folie wird nun auf die Elektrolytoberfläche gelegt und die noch freie Mittelfläche mit der Spannung oxydiert, die zur gewünschten Dicke führt (nach W a l k e n h or s t 4 $13,7 \AA /$ Volt in 3-proz. Ammoniumcitrat). Die Stromzuführung erfolgt an einer etwas aufgebogenen Seite. Der zweiseitig oxydierte Rand verhindert ein Überlaufen der Elektrolytflüssigkeit über die Rückseite des Aluminiumblättchens. Nach beendeter Oxydation befestigt man die Folie mit dünnem lackiertem Kupferdraht auf einem Rahmen aus säurefestem Material (1 mm starkes Pertinax), der etwas größer als der zweiseitig oxydierte Rand sein soll. Zum Ablösen des Aluminiums wird der Rahmen senkrecht in rauchende reine Salzsäure gestellt, der einige Kristalle Kupferchlorid beigegeben werden. Salzsäure hat gegenüber Sublimat, das Walkenhorst zum Ablösen benützt, den Vorzug, daß sie das Aluminium auf der ganzen Fläche und nicht nur von den Rändern aus angreift. Außerdem greift rauchende Salzsäure den zweiseitig oxydierten Rand nur sehr langsam an, denn es wurde festgestellt, daß etwa vorhandene Poren in der Oxydschicht durch das in Säure hoher Chlorionenkonzentration nicht lösliche $\mathrm{AlCl}_{3}$ verstopft werden. Das Aluminium cles Mittel-

* Der Rhein is chen B lat tm e t a ll AG. Grevenbroich und dem Alumini u - Wa l zwerk in Singen danke ich für die Freundlichkeit, mit der sie uns Aluminiumfolien ihrer Fertigung zur Verfügung gestellt haben. 